\title{
e-Migrinter
}

$20 \mid 2020$

Hospitalité et migration

\section{Hospitalité et migration : expériences et formes multiples de l'accueil}

Jordan Pinel

\section{CpenEdition}

Journals

Édition électronique

URL : https://journals.openedition.org/e-migrinter/2258

DOI : 10.4000/e-migrinter.2258

ISSN : 1961-9685

Éditeur

UMR 7301 - Migrinter

Référence électronique

Jordan Pinel, « Hospitalité et migration : expériences et formes multiples de l'accueil », e-Migrinter [En ligne], 20 | 2020, mis en ligne le 23 juillet 2020, consulté le 20 mai 2021. URL : http://

journals.openedition.org/e-migrinter/2258; DOI : https://doi.org/10.4000/e-migrinter.2258

Ce document a été généré automatiquement le 20 mai 2021.

Tous droits réservés 


\title{
Hospitalité et migration : expériences et formes multiples de l'accueil
}

\author{
Jordan Pinel
}

1 Alors que le contexte migratoire contemporain est souvent dépeint comme une " crise », plusieurs publications scientifiques récentes montrent surtout une crise du principe de l'hospitalité (Agier, 2018 ; Boudou, 2017 ; Brugère, Le Blanc, 2017 ; FiddianQasmiyeh, Berg, 2018). Cette littérature a confirmé l'intérêt de ce concept pour lire une diversité d'initiatives liées au contexte migratoire. Face au constat d'inhospitalité de la part de l'État dans la gestion de l'accueil, des initiatives citoyennes émergent. Qu'elles soient individuelles ou collectives, nous avons choisi de les mettre en avant dans ce dossier thématique.

2 Ce numéro a pris racine avec la journée d'étude «Expériences de l'(in)hospitalité » organisée le 1er février 2018 par Céline Bergeon ${ }^{1}$, Marie-Françoise Valette ${ }^{2}$ et Jordan Pinel, au sein du laboratoire MIGRINTER (UMR 7301). Les parutions récentes sur l'hospitalité et les migrations précédemment citées nous avaient alors inspirés et confortés dans notre démarche orientée spécifiquement vers le terrain. Notre ambition était d'ancrer les échanges de cette journée dans le registre des témoignages, qui, par leur extrême diversité permettaient des échanges de points de vue entre citoyens engagés, représentants d'associations, élus et agents de collectivités territoriales. À travers ces témoignages de proximité, nous cherchions alors peut-être inconsciemment des raisons de conserver de l'optimisme. Les échelles nationales et internationales auxquelles Mireille Delmas-Marty fait référence dans son vigoureux appel à «Faire de l'hospitalité un principe $»^{3}$ demeurent probablement pour un certain temps, moins propices à cet état d'esprit d'optimisme. Comme elle le rappelle dans sa tribune,

« Ni l'Union ni ses États membres n'ont ratifié la convention de 1990 sur les droits de tous les travailleurs migrants et de leur famille, préférant créer l'agence Frontex pour garder les frontières et adopter la "directive retour» autorisant une rétention de six mois (jusqu'à dix-huit dans certains cas). En guise de réponse à la «crise » des migrations, on entend surtout dire que la montée en puissance des 
courants populistes, aux États-Unis comme en Europe, et tout récemment en Italie, devrait conforter le choix sécuritaire, qui domine d'ailleurs le projet de loi français "pour une immigration maitrisée et un droit d'asile effectif» en débat au Parlement.».

3 Dans la lignée de la journée d'étude de 2018, les articles de ce dossier proposent aujourd'hui de rendre compte des expériences et des formes multiples de l'hospitalité dans le cadre des migrations internationales. En complémentarité avec les articles scientifiques, des témoignages d'acteurs associatifs de l'hospitalité y sont adossés et permettent d'illustrer les dispositifs d'accueil mis en place au niveau local.

Le premier article de Julie Leblanc et Jordan Pinel rappelle des pratiques inhospitalières des institutions envers les chibanis et les chibanias, ces immigrés venus en France souvent dans le cadre de dispositifs migratoires étatiques à partir des années 1960 . Aujourd'hui âgés, certains d'entre elles et eux font face à des conditions de vie précaires et des droits sociaux très limités. Dans bien des cas ce sont les acteurs locaux de l'hospitalité, souvent associatifs, qui se mobilisent pour contrebalancer des situations perçues comme injustes. L'originalité est que ces acteurs de la société civile sont mobilisés « ici » et « là-bas ", c'est-à-dire autant dans le pays d'accueil que celui d'origine rappelant la vie « d'entre-deux » de ces populations vieillissantes.

5 L'article d'Évangeline Masson Diez évoque un tout autre âge de la vie avec le cas de l'hébergement des mineurs isolés étrangers en Île-de-France. Dans cet article, c'est la relation entre les hôtes qui est analysée. Cette forme d'hospitalité privée, individuelle ou familiale, paraît simple - il s'agit d'offrir un toit à un jeune - mais relève en fait d'une multitude de pratiques et de discours qui mêlent souvent engagement et intimité du «chez-soi ». Là, c'est un hébergement en milieu urbain qui est évoqué mais où les pratiques d'hospitalité résonnent avec celles décrites en milieu rural dans l'article d'Élise Martin. Elle y étudie un système de solidarité présent dans plusieurs villages des Cévennes qui montre les liens entre engagements individuels et collectifs dans l'accueil. Des structures sociales, associatives et religieuses de ces territoires ruraux permettent des interconnexions militantes qui répondent au besoin d'assistance de certains migrants.

6 Au-delà des associations et dans un périmètre plus large, Thomas Lacroix évoque le positionnement de certaines villes qui se mobilisent dans l'accueil des demandeurs d'asile. Des réseaux de villes émergent alors - les villes sanctuaires en Grande-Bretagne ou l'Association Nationale des Villes et Territoires Accueillants en France par exemple mettant en avant un positionnement et des pratiques d'accueil allant parfois à l'encontre de certains gouvernements européens. Nationaux ou transnationaux, ces réseaux montrent des pratiques multiples car les enjeux le sont tout autant, mais également car les réalités étatiques sont différentes et laissent plus ou moins de marges aux acteurs territoriaux dans la gestion migratoire et notamment dans l'accueil.

7 Enfin, ces dernières années ont montré que le principe d'hospitalité appliqué par certains citoyens peut être perçu comme allant à l'encontre des politiques migratoires de l'État, qui par la logique de contrôle stricte des frontières et des flux migratoires se trouvent être inhospitalières. C'est ce qui a créé le "délit d'hospitalité » en 1995 (Brugère et Le Blanc, 2017) et la " pénalisation de l'hospitalité » (Saas, 2001). Le terme de « délit de solidarité » a été plus popularisé par les milieux associatifs, les défenseurs des droits ou les médias, notamment après plusieurs affaires judiciaires rendues célèbres - le procès de Cédric Herrou ou celui des «sept de Briançon" par exemple. 
Kelly Loiseau propose dans le dernier article de ce dossier de retracer la bataille judiciaire et ses enjeux, qui ont eu lieu autour du principe de l'accueil inconditionnel et solidaire. Ce dernier a été et est sûrement encore en proie à des logiques politiques étatiques plus larges où les principes d'hospitalité et de fraternité peuvent faire basculer des citoyens solidaires en « délinquants solidaires ».

Nous avons fait le choix de mettre en perspectives les articles issus de travaux scientifiques précédemment cités avec des témoignages d'acteurs de l'hospitalité. Ainsi, quatre associations locales ont accepté de témoigner dans ce dossier. Elles viennent illustrer, dans un format d'écriture libre, avec leurs mots et leurs points de vue, le principe d'hospitalité que nous retrouvons analysé par la recherche. Trois d'entre elles, basées à Poitiers, sont des témoignages de proximité géographique pour le laboratoire MIGRINTER. Dominique Royoux, président du « Toit du Monde » évoque les missions et défis auxquels est confrontée l'association présente dans le paysage pictavien depuis 1982. Plus récente, l'association « Min' de Rien » créée en 2016 est évoquée par l'une de ses fondatrices, Martine Brouillette. L'association s'est donnée pour mission l'accueil citoyen des mineurs isolés et en particulier les « mijeurs ", jeunes exclus des services de protection de l'Enfance car considérés majeurs. "Welcome Poitiers ", également créée en 2016, donne un aperçu complémentaire des pratiques citoyennes d'hospitalité mises en œuvre ces dernières années. L'association vise à accueillir des demandeurs d'asile en attente de solutions d'hébergement par l'État dans des familles d'accueil bénévoles, venant ainsi pallier une défaillance des missions de protection étatique. L'une de ses membres, Nelly Brimault, évoque avec ses mots et son ressenti les raisons de son engagement au sein de l'association. Elle tente de répondre finalement au "pourquoi s'engager?", premier pas vers les pratiques de solidarités individuelles, puis collectives qui sont visibles dans toutes ces associations. Enfin, la quatrième et dernière association - Terres d'Ancrages - est basée à Lyon depuis 2016 et accompagne des personnes exilées au quotidien en visant essentiellement la création de liens sociaux. Élise Martin, qui a proposé ce témoignage suite à ses entretiens avec deux référentes de l'association, permet ainsi de rendre compte des pratiques d'hospitalité associatives dans le contexte d'une métropole française.

9 Le constat à ce jour est le suivant : ni à l'échelle régionale, ni à l'échelle nationale, une obligation d'hospitalité publique inconditionnelle n'existe ou n'émerge. À supposer qu'arrive un jour l'heure d'un droit inconditionnel à bénéficier de l'hospitalité, elle n'est pas encore perceptible ni à court terme, ni à moyen terme. "Les lois de l'inhospitalité » (Fassin, Morice, Quiminal, 1997) continuent à caractériser le droit positif, si l'on considère que l'hospitalité ne peut être qu'inconditionnelle. Mais les pratiques de l'hospitalité existent, en France et ailleurs, en particulier en lien avec les migrations internationales. Voilà pourquoi nous avons choisi dans ce dossier de retenir des échelles et des acteurs différents, illustrant à la fois la diversité des pratiques, mais aussi des approches plus ordinaires de la question, pour interroger nos sociétés à l'aune de leur hospitalité (Berthomière, 2017). 


\section{BIBLIOGRAPHIE}

Agier, Michel (2018) L'étranger qui vient : repenser l'hospitalité, Paris, Seuil, 145 p.

Berthomière, William (2017) Les espaces de l'hospitalité, fonction-miroir de sociétés se pensant assiégées, Revue Science and Video, varia, réf. Du 02 juillet 2020 [Disponible sur Internet].

Boudou, Benjamin (2017) Politique de l'hospitalité, Paris, CNRS Éditions, 248 p.

Brugère, Fabienne ; Le Blanc, Guillaume (2017) La fin de l'hospitalité. Lampedusa, Lesbos, Calais... Jusqu'où irons-nous ?, Paris, Flammarion, 240 p.

Fassin, Didier ; Morice, Alain ; Quiminal, Catherine (1997) Les lois de l'inhospitalité, Paris, La Découverte, 278 p.

Fiddian-Qasmiyeh, Elena; Berg, Mette Louise (2018) Hospitality and Hostility Towards Migrants: Global Perspectives, Migration and Society, vol. 1, n 1, pp. V-VII.

Saas, Claire (2001) La pénalisation de l'hospitalité, Plein droit, n 49, pp. 28-31.

\section{NOTES}

1. Maître de conférences en géographie, Université de Poitiers, MIGRINTER (UMR 7301).

2. Maître de conférences en droit public, Université de Poitiers, MIGRINTER (UMR 7301).

3. Tribune du 12 avril 2018 : https://www.lemonde.fr/idees/article/2018/04/12/migrants-fairede-l-hospitalite-un-principe_5284526_3232.html

\section{AUTEUR}

JORDAN PINEL

Doctorant en géographie, Laboratoire MIGRINTER (UMR 7301) CNRS / Université de Poitiers jordan.pinel@univ-poitiers.fr 